Southampton, UK; ${ }^{2}$ Department of Radiology, Southampton University Hospital Trust, Southampton, UK; ${ }^{3}$ Department of Public Health, Southampton University Hospital Trust, Southampton, UK; ${ }^{4} H P B$ Surgery, Southampton University Hospital Trust, Southampton, UK

Introduction Treatment protocols for hepatocellular carcinoma (HCC) are evolving rapidly. We sought to determine long-term outcomes in patients with HCC treated with DEB-TACE as stand alone or part of multimodality treatment at a single centre.

Methods Our departmental database of HCC, diagnosed using EASL radiological criteria, was reviewed retrospectively. From August 2006 to January 2011, 80 patients (60 males/20 females) underwent DEBTACE some of which also had surgery and/or percutaneous ablation. A total of 186 episodes of DEB-TACE treatment were performed (minimum 1 episode and maximum 8 episodes per patient). $37.5 \%$ of patients presented with multifocal disease, a further $7.5 \%$ presented with tumour plus satellites. The mean MELD (model for end-stage liver disease) in this cohort was 9.53 (range 6-22). Demographics, procedural details, clinical measures and outcomes were studied. Median age was 69 years (range 33-87). All patients were included in the survival analysis. Overall survival was described using Kaplan-Meier methods.

Results 53 patients with a median tumour size of $49 \mathrm{~mm}$ (range $12-163 \mathrm{~mm}$ ) were treated with DEB-TACE alone with a mean number of procedures of 2.1. Here median survival was 28.5 months (798 days). The 1 - and 3-year survival rates in this group where $66 \%$ and $38 \%$ respectively. 27 patients (median tumour size of $40 \mathrm{~mm}$ (range 12-100 $\mathrm{mm}$ )) were treated with a combination of therapies and at 55 months (1540 days) the median survival had not been reached. Survival in this group was $51.1 \%$ at time data collection. In this group the 1- and 3-year survival rates were $86 \%$ and $64 \%$ respectively. In our cohort of patients DEB-TACE both with or without combination therapies, resulted in median survival of 44 months. Overall survival rates at 1 - and 3 -years were $74.5 \%$ and $50.3 \%$ respectively. There were no deaths at 30 days following a DEB-TACE episode.

Conclusion In our centre this procedure is safe and well tolerated with multimodality treatment showing an improved survival outcome. Our results highlight the importance of a multidisciplinary approach with the application of multimodal therapy in the management of HCC with an improved survival for appropriately selected patients.

Competing interests None declared.

\section{PTU-024 A "REAL WORLD" CONTROLLED STUDY OF LIVER STIFFNESS MEASURED BY ARFI (ACOUSTIC RADIATION FORCE IMPULSE) ELASTOGRAPHY IN HOSPITALISED PATIENTS WITH DECOMPENSATED ALCOHOLIC LIVER DISEASE (ALD): A NEW PARADIGM IN ASSESSMENT OF SEVERITY AND PROGNOSIS?}

doi:10.1136/gutjnl-2012-302514c.24

${ }^{1} \mathrm{D}$ I Sherman, ${ }^{*}{ }^{1} \mathrm{~K}$ Kandiah, ${ }^{2} \mathrm{M}$ Jagtiani, ${ }^{1} \mathrm{~A}$ Sharif, ${ }^{2} \mathrm{P}$ Shorvon. ${ }^{1}$ Gastroenterology Centre, NW London Hospitals NHS Trust, London, UK; ${ }^{2}$ Department of Radiology, Central Middlesex Hospital, NW London Hospitals NHS Trust, London, UK

Introduction Acoustic Radiation Force Impulse (ARFI, Virtual Touch $^{\odot}$ ) elastography is a novel validated technique for measuring liver stiffness (LS), with advantages over transient elastography including greater accuracy in ascites or obesity. However, elastography has not been well studied in acutely ill patients with decompensated chronic liver disease (CLD). We report our experience in a consecutive controlled cohort in a secondary care setting. Aims: (1) To establish whether LS is significantly different in patients hospitalised for decompensated CLD from outpatient controls with proven cirrhosis; (2) To investigate correlation between ARFI and severity scores such as DF, GAH, Lille, Child Pugh and MELD

Methods ARFI was performed by a single radiologist, using a standard 10 observation technique. 108 patients were studied: (1) 60 hospitalised patients (13 AAH-acute alcoholic hepatitis with Bili >80; 19 DALD-decompensated ALD; 12 DCLD-decompensated CLD; 10 ALC-alcoholics without severe liver disease; 6 acute hepatitis), representing $39 \%$ of 152 consecutive cases seen by the liver service; and (2) 48 age and sex matched CLD controls (all never hospitalised, 33 with biopsy-proven advanced fibrosis/cirrhosis; 15 with clear clinical/radiological/endoscopic evidence of advanced CLD).

Results Validation: technical ARFI failure $<5 \%$, IOR/median $<0.5$ in $93 \%$. Compared with CLD controls, significantly higher mean shear velocity (SV) was seen in both (a) all 32 decompensated ALD patients (AAH+ALD-2.9 \pm 0.8 vs $2.4 \pm 0.8 \mathrm{~m} / \mathrm{s}, 99 \%$ CI 0.2 to 1.0 , $\mathrm{p}=0.001$ ) and (b) all 44 decompensated CLD patients (AAH+DALD $+\mathrm{DCLD}-2.8 \pm 0.8$ vs $2.4 \pm 0.8 \mathrm{~m} / \mathrm{s}, 99 \%$ CI 0.0 to $0.8, \mathrm{p}=0.006)$. In hospitalised patients with ALD (AAH+DALD+ALC) significant correlations were seen between mean SV and both DF $(r=0.55$, $\mathrm{p}<0.001)$ and GAH $(\mathrm{r}=0.38, \mathrm{p}=0.01)$, but not with Lille score Strong correlations were shown in all inpatients between SV and Child-Pugh score $(r=0.52, p<0.001)$, and also with MELD score $(\mathrm{r}=0.42, \mathrm{p}=0.002)$, but not in controls.

Conclusion In this "real world" study, ARFI elastography is an accurate and highly reproducible tool in assessing severity and prognosis in acutely ill patients with decompensated CLD, as shown by (a) increased LS in hospitalised ALD/CLD patients compared with cirrhotic controls, and importantly (b) further increases in LS reflect severity and adverse prognosis as shown by standard scores. As higher LS scores appear to reflect disease processes beyond fibrosis, the usefulness of this "real" measurement as an alternative to current "surrogate" prognostic markers merits further analysis in larger studies.

Competing interests None declared.

\section{PTU-025 A NEW PARADIGM IN THE NON-INVASIVE ASSESSMENT OF PORTAL HYPERTENSION: A SINGLE ULTRASOUND PROCEDURE INCORPORATING ACOUSTIC RADIATION FORCE IMPULSE (ARFI) ELASTOGRAPHY PREDICTS OVER 90\% OF OESOPHAGO-GASTRIC VARICES AND PORTAL GASTROPATHY}

doi:10.1136/gutjnl-2012-302514c.25

${ }^{1} \mathrm{D}$ । Sherman, ${ }^{*}{ }^{1} \mathrm{~K}$ Kandiah, ${ }^{*}{ }^{2} \mathrm{M}$ Jagtiani, ${ }^{1} \mathrm{~A}$ Sharif, ${ }^{2} \mathrm{P}$ Bassett, ${ }^{2} \mathrm{P}$ Shorvon. ${ }^{1}$ Gastroenterology Centre, NW London Hospitals NHS Trust, London, UK; ${ }^{2}$ Department of Radiology, Central Middlesex Hospital, NW London Hospitals NHS Trust, London, UK

Introduction Accurate non-invasive techniques for imaging portal hypertension (PHT) have long been the goal of hepatologists. However the sensitivity of standard ultrasound (US) alone is too low to achieve this. The combined use of elastography with US to detect PHT has not been investigated. ARFI (Virtual Touch ${ }^{\circledR}$ ) elastography is a novel validated technique for measuring liver stiffness (LS), with practical advantages over transient elastography including real-time scanning. We report our experience of this technique in assessing PHT in a consecutive secondary care cohort. Aims To establish whether the addition of LS estimation by ARFI to conventional US Doppler assessment of the spleno-portal venous system can reliably predict the presence of significant endoscopic PHT.

Methods Conventional US and elastography were performed simultaneously at one session by a single radiologist, using a Siemens Acuson $52000^{\mathrm{TM}}$ and $4 \mathrm{C} 1$ probe $4 \mathrm{MHz}$ transducer and a 
standard, validated 10 observation right lobe ARFI technique. Within the study period, 82/108 chronic liver disease (CLD) patients (60 hospitalised and 48 outpatients) underwent both elastography and upper GI endoscopy. Standard US data collected included spleen size, portal vein Doppler flow velocity, direction and waveform, presence of collaterals, and platelet count.

Results Significant endoscopic PHT, defined as oesophageal/gastric varices and/or moderate to severe portal hypertensive gastropathy, was seen in $34 / 82$ patients (41\%). Median LS measured by shear velocity was significantly higher in the PHT group (2.9 vs $2.2 \mathrm{~m} / \mathrm{s}$, $\mathrm{p}<0.001)$. A 10 point "SPVD" (spleen-platelets/portal vein Doppler) scoring system, devised to include all US parameters and platelet count, showed significantly higher median scores in PHT (3 vs 1, $\mathrm{p}<0.001)$. Multiple logistic regression analysis demonstrated that both ARFI and SPVD score were independent predictors of PHT (OR, 95\% CI $2.73,1.22$ to $6.13, \mathrm{p}=0.02$; and $3.08,1.89$ to $5.02, \mathrm{p}<0.001$, respectively). AUROC analysis showed that a best fit combination of ARFI+SPVD score showed the highest overall predictive value at 0.91 , compared with ARFI or SPVD alone (0.72 and 0.87, respectively).

Conclusion In this retrospective "real world" study the addition of ARFI to standard US parameters using a combined scoring system achieved high $(>90 \%)$ predictive value for the non-invasive detection of endoscopic PHT. A further prospective study, with refinement of US Doppler technique, is now in progress to confirm these promising results. Single session ARFI+US may accurately guide PHT diagnosis and selection for endoscopic surveillance in CLD.

Competing interests None declared.

\section{PTU-026 PREVALENCE OF LIVER HISTOLOGICAL ABNORMALITIES IN TYPE 1 DIABETES AND THE LONG TERM CONSEQUENCES}

doi:10.1136/gutjnl-2012-302514c.26

${ }^{1} \mathrm{D}$ J Harman, ${ }^{*}{ }^{2} \mathrm{R}$ Harris, ${ }^{3} \mathrm{~A}$ Gazis, ${ }^{4} \mathrm{P}$ Kaye, ${ }^{1} \mathrm{G}$ Aithal. ${ }^{1}$ Hepatology, NIHR BRU, Nottingham, UK; ${ }^{2}$ General Medicine, Nottingham University Hospitals NHS Trust, Nottingham, UK; ${ }^{3}$ Endocrinology, Nottingham University Hospitals NHS Trust, Nottingham, UK; ${ }^{4}$ Histopathology, Nottingham University Hospitals NHS Trust, Nottingham, UK

Introduction Patients with type 1 diabetes have a higher prevalence of raised liver enzymes than the general population. Ultrasound diagnosis of non-alcoholic fatty liver disease has been reported to be common in type 1 diabetes despite associated insulin deficiency rather than insulin resistance. However, the histological spectrum of liver disease in type 1 diabetes and the natural history of chronic liver disease in this cohort is unknown. We describe the histological findings of patients with type 1 diabetes who had liver biopsy in a tertiary referral centre, and their long-term clinical outcome.

Methods The DIAMOND database, which contains longitudinal data for over $95 \%$ of type 1 diabetes patients from an overall catchment population of 750000 in Nottingham, was crossmatched with the clinical pathology database to identify those who had undergone liver biopsy. Case notes were reviewed to obtain followup data, and identify liver and non-liver related outcomes.

Results Out of 2800 patients with Type 1 Diabetes, 57 patients underwent a total of 82 liver biopsies. Common indications for biopsy were abnormal liver enzymes (28 patients), malignancy (8), Hepatitis C staging (7) and clinical evidence of cirrhosis (3). On index biopsy, $86 \%$ had significant histological abnormalities (Abstract PTU-026 figure 1) and 10 patients (17.5\%) had cirrhosis. During a total follow-up of 336.4 patient years (median 5.6 years), a further four patients developed cirrhosis, giving a cirrhosis prevalence of at least 500 per 100000 population - this compares with an estimated UK cirrhosis prevalence of 76.3 per 100000 population. ${ }^{1}$ Portal hypertensive sequelae occurred in 11 patients (78.6\%) with cirrhosis and hepatocellular carcinoma in three patients. 22 patients (38.6\%) died during follow-up. Crude death rate was 6539 per 100000 person years, compared with national Type 1 Diabetes data ${ }^{2}$ of 1878 per 100000 person years.

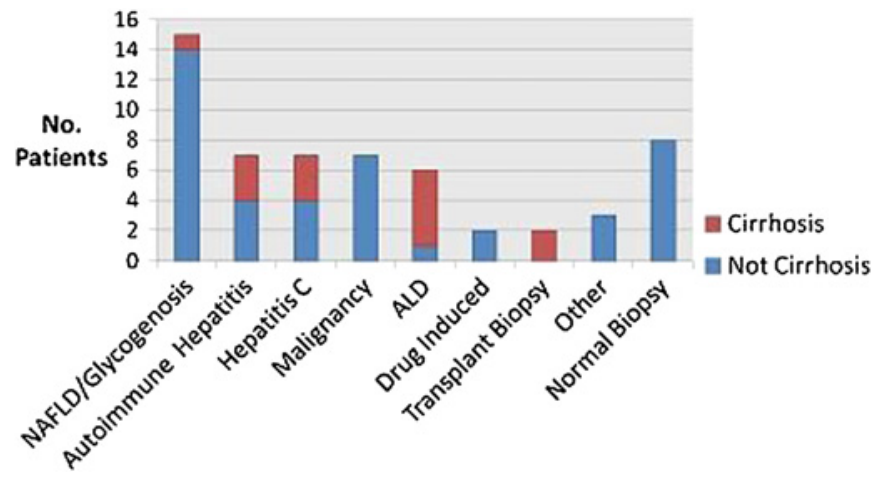

Abstract PTU-026 Figure 1 Index liver biopsy diagnoses.

Conclusion Type 1 Diabetes is associated with significant liver histology abnormalities and a higher than expected occurrence of cirrhosis, portal hypertension and mortality. These findings have implications for long-term management of patients with Type 1 Diabetes.

Competing interests None declared.

\section{REFERENCES}

1. Fleming $\mathbf{K M}$, Aithal GP, Solaymani-Dodaran $\mathbf{M}$, et al. Incidence and prevalence of cirrhosis in the United Kingdom, 1992-2001: a general population-based study. J Hepatol 2008;49:732-8.

2. NHS IC. National Diabetes Audit Mortality Analysis 2007-2008. NHS IC, 2011

\section{PTU-027 LIFETIME ALCOHOL CONSUMPTION IN HEAVY DRINKERS WITH AND WITHOUT LIVER DISEASE. 1: THRESHOLD EFFECT AND MALE-FEMALE DIFFERENCES}

doi:10.1136/gutjnl-2012-302514c.27

${ }^{1} \mathrm{D}$ Gleeson, ${ }^{*} \mathrm{~A}$ Ali, ${ }^{1} \mathrm{~J}$ Jones, ${ }^{1} \mathrm{M}$ Bradley, ${ }^{2} \mathrm{R}$ Peck, ${ }^{3} \mathrm{~K}$ McCormack. ${ }^{1}$ Liver Unit, Sheffield Teaching Hospitals, Sheffield, UK; ${ }^{2}$ Radiology, Sheffield Teaching Hospitals, Sheffield, UK; ${ }^{3}$ Research, Sheffield Teaching Hospitals, Sheffield, UK

Introduction It is unclear whether the risk of alcoholic liver disease (ALD) is related to amount of alcohol consumed (dose effect) or is independent of alcohol consumption above a given threshold (J Hepatol 2004;41:25). The perception that women are more susceptible to ALD than men is based partly on observations that alcohol consumption in women with ALD is less than that of men with ALD. We have previously (Am J Gastro 2008;103:3039) characterised two large cohorts of heavy drinkers ( $>60$ Units/wk (M) or $>40$ Units/wk (F) for $>5$ years): patients with decompensated ALD and controls without serious liver disease on clinical, laboratory and ultrasound examination. Here, we aimed to compare total lifetime alcohol consumption (TLA) between these cohorts and to examine male-female differences in consumption.

Methods Subjects (328 patients, 233 male, mean age $48 \mathrm{yr}$ and 237 heavy-drinking controls, 187 male, mean age $48 \mathrm{yr}$ ) completed a lifetime alcohol questionnaire. Total alcohol consumption was calculated and the predominant beverage group recorded, separately, at home and outside home, and during Monday-Thursday and Friday-Sunday. Data were summed over each stable drinking period in the subject's lifetime. For individual beverage analysis, we assumed that all alcohol consumed in a given circumstance was the 